\title{
Occurrence and Characterization of Dimethachlon Insensitivity in Sclerotinia sclerotiorum in Jiangsu Province of China
}

\author{
Hui-Xia Ma, Xi-Jie Feng, Yu Chen, Chang-Jun Chen, and Ming-Guo Zhou, College of Plant Protection, Nanjing \\ Agricultural University, Nanjing, 210095, China
}

\begin{abstract}
Ma, H.-X., Feng, X.-J., Chen, Y., Chen, C.-J., and Zhou, M.-G. 2009. Occurrence and characterization of dimethachlon insensitivity in Sclerotinia sclerotiorum in Jiangsu Province of China. Plant Dis. 93:36-42.

Sclerotinia stem rot, caused by Sclerotinia sclerotiorum, is the main fungal disease of oilseed rape (Brassica napus) in China. Numerous fungicide applications are required for control. Dimethachlon, one of the dicarboximide fungicides, has been the major fungicide for disease control after benzimidazole resistance became widespread. Fungal populations were collected throughout Jiangsu Province between 2006 and 2007 in order to determine their sensitivity to dimethachlon. A total of 1,066 single-sclerotium isolates of S. sclerotiorum were collected, and most of the isolates were considered sensitive to dimethachlon. Five isolates collected in Yancheng and Changzhou showed normal growth at $5 \mu \mathrm{g} / \mathrm{ml}$ dimethachlon with the resistance factor $\approx 10$ (resistance factor was estimated as ratios between the $\mathrm{EC}_{50}$ values of resistant isolates and the average $\mathrm{EC}_{50}$ values of sensitive ones) compared to the sensitive isolates $\left(\mathrm{EC}_{50}\right.$ is the concentration of fungicide causing $50 \%$ reduction in growth). Through in vitro selection for resistance to the fungicide, 25 dimethachlon-resistant mutants were derived from 10 wild-type isolates of S. sclerotiorum. The resistance factors for the isolates ranged from 198 to 484, and the isolates were considered highly resistant to dimethachlon. Therefore, at least two different mechanisms of resistance seem to be involved: one that may provide a moderate resistance (insensitivity) and a second that may give a high resistance level under laboratory conditions. There was positive cross-resistance between dimethachlon and other dicarboximide fungicides, such as iprodione and procymidone, in these $S$. sclerotiorum isolates. The field dimethachlon-insensitive and the laboratory-induced dimethachlon-resistant isolates appeared to have mycelial growth, sclerotial production, and pathogenicity comparable to their wild-type parental isolates. Also, results of osmotic tests showed that there were no significant difference in mycelial radial growth between the field dimethachlon-sensitive and field dimethachlon-insensitive isolates on potato dextrose agar plates amended with $2,4,6$, or $8 \%(\mathrm{wt} / \mathrm{vol}) \mathrm{NaCl}$, but the laboratoryinduced dimethachlon-resistant isolates grew significantly more slowly than their wild-type sensitive parents under all concentrations of $\mathrm{NaCl}$. Because these studies yielded a high frequency of laboratory resistance in S. sclerotiorum, together with the occurrence of field insensitivity, appropriate precautions against resistance development in natural populations should be taken.
\end{abstract}

Sclerotinia sclerotiorum (Lib.) de Bary is a cosmopolitan fungal pathogen that attacks more than 400 species of higher plants (7), including crops such as soybean, bean, sunflower, canola, and oilseed rape (Brassica napus L.) (37). Infection can occur on leaves, stems, and pods at different developmental stages $(1,14,48)$. The disease is important in many countries, including Canada (4), the United

Corresponding author: Ming-Guo Zhou

E-mail: mgzhou@njau.edu.cn

H.-X. Ma, X.-J. Feng, and Y. Chen share joint first authorship.

Accepted for publication 18 September 2008.

doi:10.1094/PDIS-93-1-0036

This article is in the public domain and not copyrightable. It may be freely reprinted with customary crediting of the source. The American Phytopathological Society, 2009.
States (8,37), Australia (24), and China (51). For example, Sclerotinia stem rot in China causes yield loss ranging from 10 to $80 \%$, and oil quality declines as well (3). Oilseed rape is the major oilseed crop in China, with approximately $70 \times 10^{6}$ ha in production (50). Jiangsu Province is the main production area, where oilseed rape is planted on about 700,000 ha every year.

Because breeding programs for disease resistance have been hampered by limited gene sources (28), Sclerotinia stem rot continues to impose serious limitations on oilseed rape production all over the world. In practice, application of fungicides is the principal tool in most oilseed rape crops for managing Sclerotinia stem rot. A benzimidazole fungicide, carbendazim (MBC), was routinely used to control this disease during the 1980s in China, but widespread control failures attributed to development of MBC resistance in $S$. sclerotiorum were reported in 2001 and $2002(34,41,49)$.
The dicarboximide fungicides (e.g., iprodione, procymidone, and vinclozolin) have also been used for control of plant diseases incited by $S$. sclerotiorum, $S$. homoeocarpa, and $S$. minor for many years $(9,19,21,32,33,44,45)$, although modes of action and resistance mechanisms of plant pathogens to these compounds are not yet well known. Dimethachlon ( $N$-(3,5dichlorophenyl)-succinimide, Ohric, Wenzhou Pesticide Factory, Zhejiang Province, China), a dicarboximide fungicide effective against various plant pathogenic fungi (36), has also been widely used for the control of Sclerotinia stem rot in China for more than 10 years, at an application rate of one to two sprays per season $(26,42)$. However, it appears that repeated applications of these fungicides have caused the emergence of resistant strains of several plant pathogens, including $S$. homoeocarpa and $S$. minor $(21,45)$, in other countries $(5,22,23,30)$. Thus, it is necessary to incorporate effective antiresistance strategies when dicarboximide fungicides are extensively used to control plant diseases (10).

Previous studies also reported that high levels of dicarboximide resistance could be induced in the laboratory and that the laboratory-induced dicarboximide-resistant isolates exhibited greater osmotic sensitivity in several fungi with decreased virulence, while field isolates with dicarboximide resistance did not exhibit changed osmotic sensitivity and retained high virulence on plants $(12,13,18,30)$, suggesting that the field-resistant isolates might have sufficient parasitic fitness to compete with field-sensitive isolates.

No information is available on dimethachlon sensitivity in field populations of $S$. sclerotiorum in China. The objectives of this study were to: (i) characterize dimethachlon sensitivity in several oilseed rape growing regions in Jiangsu Province; (ii) recover laboratory-resistant mutants of $S$. sclerotiorum to dimethachlon and investigate resistance stability in both the laboratory-induced dimethachlon-resistant isolates and the field dimethachlon-resistant isolates; (iii) explore the cross-resistance patterns between dimethachlon and other well-known dicarboximide fungicides, such as iprodione and procymidone; (iv) compare the biological characteristics of the isolates collected in the field with those of isolates resistant to dimethachlon and other dicarboximide fungicides obtained by selection in the laboratory; and (v) de- 
termine the osmotic sensitivity between field isolates resistant to dimethachlon and laboratory-resistant isolates.

\section{MATERIALS AND METHODS}

Fungal pathogens. All isolates of $S$. sclerotiorum used in the study were collected from oilseed rape fields in Jiangsu Province of China from 2006 to 2007 (Fig. 1). The oilseed rape fields were separated from each other by more than $10 \mathrm{~km}$. In each field, several plants with symptoms of Sclerotinia stem rot were randomly collected from a designated $5.0 \times 5.0 \mathrm{~m}^{2}$ area, air-dried, placed in paper envelopes, and stored at $-4^{\circ} \mathrm{C}$. All isolates were derived from individual sclerotia collected from oilseed rape plants. The sclerotia were surface-sterilized in $0.1 \%$ sodium hypochlorite for $5 \mathrm{~min}$, rinsed in sterile distilled water for $30 \mathrm{~s}$, bisected, and one of the two halves was placed on a potato dextrose agar (PDA; $200 \mathrm{~g}$ potato, $20 \mathrm{~g}$ agar, and $20 \mathrm{~g}$ dextrose per liter of distilled water) plate. The plates were incubated for 3 days at $25^{\circ} \mathrm{C}$ in a growth chamber $(12 \mathrm{~h}$ photoperiod). Pure cultures were obtained by transfer of a single sclerotium and maintained on PDA slants at $4^{\circ} \mathrm{C}$ for 2 to 4 weeks $(11,25,40)$.

Fungicides. Dimethachlon in technical grade $(90.0 \%)$ was provided by Wenzhou Pesticide Factory and dissolved in methanol $(>99.5 \%)$ to $10 \mathrm{mg} / \mathrm{ml}$ for the stock solution. Iprodione and procymidone in technical grade $(96.2$ and $98.0 \%$, respectively) were provided by Jiangsu Kuaida Agrochemical Co. Ltd., China and Sumimoto Chemical Co. Ltd., Japan, respectively, and were dissolved in acetone to 10 $\mathrm{mg} / \mathrm{ml}$ for the stock solution. All stock solutions were diluted into autoclaved media which had cooled to 40 to $50^{\circ} \mathrm{C}$, and the $\mathrm{pH}$ was adjusted to 6.8 in all media using $0.1 \mathrm{~mol} / \mathrm{liter} \mathrm{HCl}$.

Resistance determination. An initial qualitative assessment (preliminary test) was carried out for all isolates. To simplify the analysis of field resistance to dimethachlon in S. sclerotiorum, only two concentrations were used: 0 and $5.0 \mu \mathrm{g} / \mathrm{ml}$. An inverted mycelial plug ( $5 \mathrm{~mm}$ in diameter) cut from the edge of a 3-day-old colony was transferred to a 9-cm petri dish containing amended or unamended PDA. After 2 days of incubation in a growth chamber at $25^{\circ} \mathrm{C}$ and 12 -h photoperiod, mycelial growth was measured. Isolates that showed normal growth at $5 \mu \mathrm{g} / \mathrm{ml}$ dimethachlon were tentatively considered resistant (scored as growth), whereas the completely inhibited ones were considered sensitive (scored as no growth) (27).

Another series of tests was made to find the concentration of the fungicide causing a $50 \%$ reduction in the growth rate compared to an unamended control $\left(\mathrm{EC}_{50}\right)$. In the $\mathrm{EC}_{50}$ assay, PDA medium was amended with six concentrations of dimethachlon: $0,0.625,1.25,2.5,5.0$, and
$10.0 \mu \mathrm{g} / \mathrm{ml}$ for all tentatively resistant isolates identified above and $0,0.125,0.25$, $0.5,1.0$, and $2.0 \mu \mathrm{g} / \mathrm{ml}$ for 152 arbitrarily selected wild-type sensitive isolates in both 2006 and 2007 (72 isolates collected in 2006 and 80 isolates collected in 2007). Inverted mycelial plugs $(5 \mathrm{~mm}$ in diameter) cut from the edges of 3-day-old colonies were transferred to $9-\mathrm{cm}$ petri dishes containing the amended media. Three replicates per concentration were used, and all tests were repeated twice. The mycelial growth was measured after 2 days of incubation at $25^{\circ} \mathrm{C}$ in a growth chamber $(12 \mathrm{~h}$ photoperiod). Two perpendicular diameters of each fungal colony were measured and averaged (the diameter of the plug was subtracted). $\mathrm{EC}_{50}$ values were determined by probit analysis (2).

Sensitivity tests to find the minimum inhibitory concentration were carried out on the sensitive isolates collected in both 2006 and 2007. The procedure followed for the test for minimum inhibitory concentration was the same as described above for the $\mathrm{EC}_{50}$ test, but different concentrations of the fungicide were used: 0 , $0.5,1.0,1.5,2.0,2.5$, and $3.0 \mu \mathrm{g} / \mathrm{ml}$ among all the sensitive isolates. The evaluation was made after 2 days of incubation at $25^{\circ} \mathrm{C}$ in a growth chamber $(12 \mathrm{~h}$ photoperiod). Fungal growth was observed, and the concentration that was able to inhibit mycelial growth completely (no mycelial growth) was recorded as the minimum inhibitory concentration. The test to find the minimum inhibitory concentration was repeated twice.

In vitro selection for resistance. To induce dimethachlon-resistance through selection, fresh mycelial plugs $(5 \mathrm{~mm}$ in diameter) from colony margins of the five randomly selected wild-type sensitive isolates were transferred to PDA plates amended with $5.0 \mu \mathrm{g} / \mathrm{ml}$ dimethachlon and incubated in the growth chamber for 3 days. Any fast-growing sectors from the otherwise restricted colonies were selected and transferred after 2 days to PDA plates amended with $5.0 \mu \mathrm{g} / \mathrm{ml}$ dimethachlon. Single-sclerotium isolates were established from dimethachlon-resistant mutants. Autoclaved PDA was amended with dimethachlon to obtain final concentrations of $15,30,60,120$, and $240 \mu \mathrm{g} / \mathrm{ml}$, and the $\mathrm{EC}_{50}$ values of resistant mutants were determined as described above.

The $\mathrm{EC}_{50}$ values allowed the calculation of the resistance factor (RF), which shows the sensitivity level of isolates. The RF was estimated as ratios between the $\mathrm{EC}_{50}$ values of resistant isolates and the average $\mathrm{EC}_{50}$ values of sensitive ones. It was calculated both on the resistant isolates collected from the field for the 2 years of the monitoring work and on the resistant mutants selected under laboratory conditions for assessment of the resistance risk.

Resistance stability. All five field dimethachlon-resistant and three laboratoryinduced dimethachlon-resistant isolates from each of their parental isolates (in all, 15 laboratory-induced dimethachlonresistant isolates) were selected randomly, and 50 single-sclerotium isolates were established for each isolate as mycelial cultures. Each of the latter was transferred to PDA plates containing $5.0 \mu \mathrm{g} / \mathrm{ml} \mathrm{di-}$ methachlon to determine whether the resistant isolates maintained dimethachlon resistance. In addition, all the resistant isolates and the three parental isolates were transferred eight times onto new fungicidefree PDA plates with three plates per isolate (2-day culture for one time) for over 16 days or stored on fungicide-free PDA slants with three slants per isolate at $4^{\circ} \mathrm{C}$ in the refrigerator for 60 days. The $\mathrm{EC}_{50}$ val-

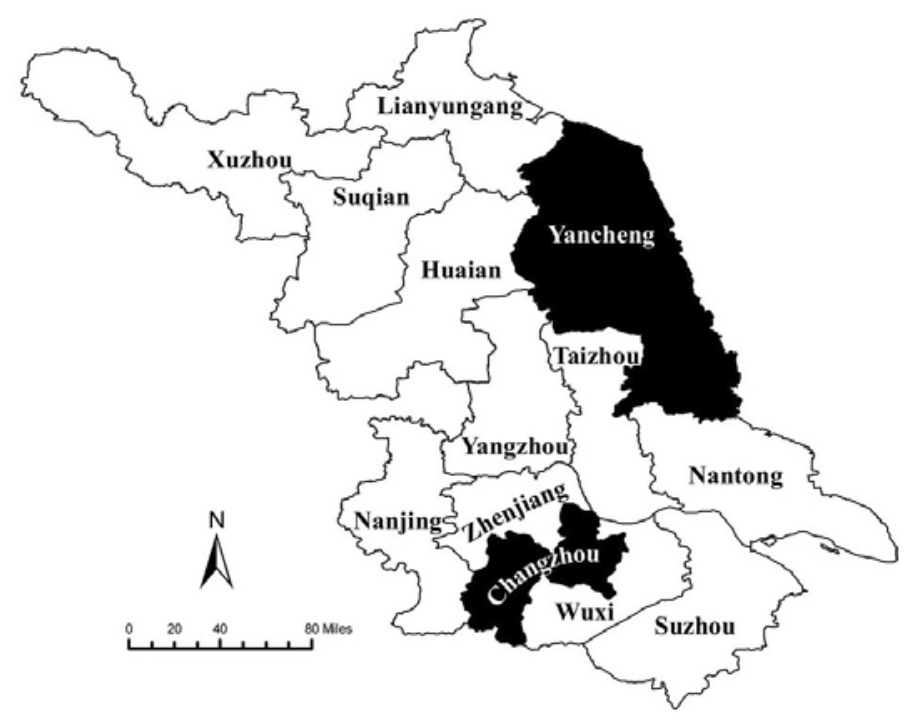

Fig. 1. Map of Jiangsu Province, China, where 1,066 isolates of Sclerotinia sclerotiorum were collected from oilseed rape plants with symptoms of Sclerotinia stem rot. The two cities shaded (Yancheng and Changzhou) indicate where field isolates with reduced sensitivity to dimethachlon were detected. 
ues were then determined again as described above to test the resistance stability. The whole test to determine resistance stability was repeated twice.

Cross-resistance to other dicarboximide fungicides. All field dimethachlonresistant isolates and six laboratoryinduced dimethachlon-resistant isolates from each of the two parental isolates were subcultured on PDA plates. Cultures were grown for 2 days, and then 5-mmdiameter mycelial plugs were transferred from the margins of the colonies onto a series of concentrations of dimethachlon-, iprodione-, and procymidione-modified PDA media in 9-cm petri dishes, and incubated at $25^{\circ} \mathrm{C}$ (Table 1). The concentrations used were: $0.125-2,0.625-10$, and $18.75-300 \mu \mathrm{g} / \mathrm{ml}$ of dimethachlon; $0.075-$ $0.4,0.625-10$, and $18.75-300 \mu \mathrm{g} / \mathrm{ml}$ of iprodione; and 0.075-0.4, 0.625-10, and $12.5-400 \mu \mathrm{g} / \mathrm{ml}$ of procymidione for the field sensitive isolates, the field dimethachlon-resistant isolates, and the laboratory-induced dimethachlon-resistant isolates, respectively (Table 1 ). The $\mathrm{EC}_{50}$ values were determined as described above, and the sensitivity of mutants to dimethachlon and the other two fungicides was compared (47). The whole test to determine the cross-resistance was repeated twice.

Mycelial growth and sclerotial production by dimethachlon-resistant isolates. All the field isolates resistant to dimethachlon and six laboratory-induced dimethachlon-resistant isolates, together with their parental isolates, were chosen for the mycelial growth and sclerotial production test. Mycelial plugs ( $5 \mathrm{~mm}$ diameter) were cut from the edge of a 3-day-old colony and placed in the center of petri dishes $(9 \mathrm{~cm}$ diameter) containing PDA. The plates were incubated at $25^{\circ} \mathrm{C}$ in a growth chamber (12 h photoperiod), and colony diameters $(\mathrm{cm})$ were measured after 2 days of incubation. Three replicates were conducted for each of the isolates, and all the tests were repeated twice. Mean colony diameter $(\mathrm{cm})$ was measured to compare mycelial growth. After 4 weeks, the numbers and the dry weight of sclerotia per plate were measured (35). Briefly, the number of sclerotia per plate was counted, the total dry weight of the sclerotia per plate was measured, and the average dry weigh of a sclerotium was calculated. In addition, a fresh mycelial plug of each isolate from colony margins was transferred into a flask containing $100 \mathrm{ml}$ of potato dextrose (PD) broth for shake culture $\left(120 \mathrm{r} / \mathrm{min}, 25^{\circ} \mathrm{C}\right)$ for 2 days. Then the mycelia were washed with distilled water three times to remove possibly contaminating medium and dried in an air drier at $80^{\circ} \mathrm{C}$ for $8 \mathrm{~h}$. The dry weight of the mycelia was measured to further compare the mycelial growth. There were three replications for each of the isolates and the test was repeated twice.

In vitro pathogenicity tests. All the selected isolates used for the mycelial growth and sclerotial production test as described above were also used for the pathogenicity tests. Leaves from healthy oilseed rape plants cv. Ningyou 16 (Jiangsu Fengqing Seed Science Company) were detached, washed with sterile distilled water, blotted with sterile filter paper to remove excess water, and air-dried. A single leaf was detached from each plant. Leaf age, growing position, and leaf size were similar for all treatments in the tests. Mycelial plugs (5 $\mathrm{mm}$ diameter) from a 3 day culture of $S$. sclerotiorum were placed on the adaxial surface of the leaf (two per leaf, either side of the midrib, avoiding major veins). Lesions were measured as the mean of two diameters measured at right angles to one another after 4 days at $22^{\circ} \mathrm{C}$ under a 16 -h photoperiod and $\approx 80 \%$ relative humidity. The lengths of the long and short axes were averaged, and the radius was used to find the area of a circle (17). The experiment was performed twice with more than seven replicates per treatment.

Determination of osmotic sensitivity. For each isolate, a mycelial plug $(5 \mathrm{~mm}$ diameter) was taken from the edge of a 3day-old colony and transferred onto PDA amended with 2, 4, 6, or $8 \%$ (wt/vol) $\mathrm{NaCl}$. Plates without $\mathrm{NaCl}$ were used as a nontreatment control. Three plates of each $\mathrm{NaCl}$ concentration were incubated at $25^{\circ} \mathrm{C}$ in a growth chamber $(12 \mathrm{~h}$ photoperiod) for 2 days. The diameter of the fungal colony was measured in millimeters for each plate (30). The percentage of the mycelial radial growth inhibition (RGI) relative to the $\mathrm{NaCl}$-free control was calculated. The experiment was repeated twice, and significant differences in colony diameter of each isolate were determined.

Statistical analysis. All analyses were conducted using SPSS 14.0 (Statistical Package for the Social Science, SPSS Inc., Chicago, IL). The $\mathrm{EC}_{50}$ of the isolates, defined as the concentration of the fungicide that caused a reduction in colony diameter of $50 \%$, was estimated by linear regression of the log of the colony diameter versus the fungicide concentration. The ANOVA procedure of SPSS and Fisher's LSD, $P=0.05$, were used to determine significant differences on the $\mathrm{EC}_{50}$ values, the biological characteristics (mycelial growth, sclerotial production, and pathogenicity), and the osmotic sensitivity in field dimethachlon-sensitive, field dimethachlon-resistant, and laboratory-induced dimethachlon-resistant isolates.

\section{RESULTS}

Dimethachlon sensitivity of field isolates. Of the 1,066 single-sclerotium isolates analyzed in the present study, only five (CZ162, CZ138, YC310, YC312, and YC334) from Changzhou and Yancheng were insensitive to $5.0 \mu \mathrm{g} / \mathrm{ml}$ dimethachlon in the preliminary test (Fig. 1, Table 2). Moreover, mycelial growth of all sensitive isolates was completely inhibited at the concentration of $2.5 \mu \mathrm{g} / \mathrm{ml}$, and thus 2.5 $\mu \mathrm{g} / \mathrm{ml}$ could be treated as the minimum inhibitory concentration value for distinguishing the sensitive and resistant isolates in this study. The $\mathrm{EC}_{50}$ values for inhibition of mycelial growth in the five field isolates insensitive to dimethachlon were 2.4 to $4.4 \mu \mathrm{g} / \mathrm{ml}$, with an average $\mathrm{EC}_{50}$ value of $3.2 \mu \mathrm{g} / \mathrm{ml}$. The average $\mathrm{EC}_{50}$ value for the 152 arbitrarily selected sensitive isolates was $0.3 \mu \mathrm{g} / \mathrm{ml}$ (Table 3). Therefore, the RF values for the five field isolates insensitive to dimethachlon were 7.1 to 13.2 , with an average $\mathrm{RF}$ value 9.4.

In vitro selection for resistance. In the selection tests, all five parental sensitive isolates grew poorly on the PDA plates amended with $5 \mu \mathrm{g} / \mathrm{ml}$ dimethachlon during the first 3 days. Only a few spontaneous sectors from the five parental isolates grew rapidly after 2 days, and consequently, 25 resistant mutants from five parental sensitive isolates were obtained by selection on the fungicide after 10 days. The $\mathrm{EC}_{50}$ values of six of the 25 mutants ranged from 68.0 to $166.4 \mu \mathrm{g} / \mathrm{ml}$ with the RF values 197.6 to 483.7 (Table 3), indicating high level of resistance.

Stability of dimethachlon resistance. All single-sclerotium progeny of insensitive field isolates and the resistant mutants grew normally on PDA amended with 5 $\mu \mathrm{g} / \mathrm{ml}$ dimethachlon through eight transfers and when stored on PDA slants at $4^{\circ} \mathrm{C}$ for 60 days (data not shown).

Cross-resistance to dicarboximide fungicides. All five dimethachlon-in-

Table 1. Concentrations $(\mu \mathrm{g} / \mathrm{ml})$ used to determine the concentration causing $50 \%$ reduction in growth rate $\left(\mathrm{EC}_{50}\right)$ values for sensitivity to dicarboximides in Sclerotinia sclerotiorum isolates collected in Jiangsu Province, China

\begin{tabular}{llll}
\hline Resistance phenotypes of isolates & Dimethachlon $(\boldsymbol{\mu g} / \mathbf{m l})$ & Iprodione $(\boldsymbol{\mu g} / \mathbf{m l})$ & Procymidione $(\boldsymbol{\mu g} / \mathbf{m l})$ \\
\hline Field dimethachlon-sensitive & $2,1,0.5,0.25,0.125$ & $0.4,0.3,0.2,0.15,0.1,0.075$ & $0.4,0.3,0.2,0.15,0.1,0.075$ \\
Field dimethachlon-insensitive & $10,5,2.5,1.25,0.625$ & $10,5,2.5,1.25,0.625$ & $10,5,2.5,1.25,0.625$ \\
Laboratory-induced dimethachlon-resistant & $300,200,150,75,37.5,18.75$ & $300,150,75,37.5,18.75$ & $400,200,100,50,25,12.5$ \\
\hline
\end{tabular}

${ }^{\mathrm{z}}$ Isolates that showed normal growth at $5 \mu \mathrm{g} / \mathrm{ml}$ dimethachlon were considered resistant, whereas the completely inhibited ones were considered sensitive. 
sensitive field isolates and six laboratoryinduced dimethachlon-resistant isolates had higher $\mathrm{EC}_{50}$ values than the field sensitive isolates on the dicarboximideamended PDA plates (Table 3). Comparison of the $\mathrm{EC}_{50}$ between the insensitive and sensitive isolates showed that there was cross-resistance between dimethachlon and other well-known dicarboximide fungicides, such as iprodione and procymidone, in both field dimethachloninsensitive and laboratory-induced dimethachlon-resistant $S$. sclerotiorum isolates (Table 3).

Mycelial radial growth and sclerotial production in vitro. The insensitive field isolates and the laboratory-induced dimethachlon-resistant isolates grew normally with thick aerial mycelium on PDA plates amended with $5 \mu \mathrm{g} / \mathrm{ml}$ dimethachlon. The growth of the field sensitive parental isolates was completely inhibited. Most of the laboratory-induced dimethachlon-resistant isolates had reduced radial growth on PDA plates compared to their parental isolates (Table 4). Sclerotial production by the insensitive field and the laboratory-induced dimethachlon-resistant isolates and their parental isolates was compared from a 28-day culture on PDA. All isolates produced sclerotia, but numbers were reduced in the resistant compared to the sensitive isolates (Table 4).

Comparison of pathogenicity in vivo. Six laboratory-induced dimethachlonresistant isolates and their parental isolates, and the 10 field-insensitive and -sensitive isolates exhibited pathogenicity on oilseed

Table 2. Number of isolates of Sclerotinia sclerotiorum sensitive and insensitive to dimethachlon found on oilseed rape growing in production areas throughout Jiangsu Province of China in 2006 and 2007

\begin{tabular}{|c|c|c|c|c|}
\hline \multirow[b]{2}{*}{ Cities } & \multirow{2}{*}{$\begin{array}{c}\text { Years of } \\
\text { dicarboximide use }\end{array}$} & \multicolumn{2}{|c|}{ Sample year ${ }^{y}$} & \multirow{2}{*}{$\begin{array}{c}\text { Overall } \\
(\text { by location })^{y}\end{array}$} \\
\hline & & 2006 & 2007 & \\
\hline Suzhou & 6 & $0(33)$ & $0(70)$ & $0(103)$ \\
\hline Taizhou & 7 & $0(96)$ & $0(70)$ & $0(166)$ \\
\hline Wuxi & 6 & None $^{\mathrm{z}}$ & $0(96)$ & $0(96)$ \\
\hline Yangzhou & 6 & $0(40)$ & $0(67)$ & $0(107)$ \\
\hline Huai’an & 7 & None & $0(47)$ & $0(47)$ \\
\hline Yancheng & 7 & $3(50)$ & $0(58)$ & $3(108)$ \\
\hline Suqian & 7 & None & $0(44)$ & $0(44)$ \\
\hline Zhenjiang & 7 & None & $0(122)$ & $0(122)$ \\
\hline Nantong & 6 & $0(122)$ & $0(89)$ & $0(201)$ \\
\hline Changzhou & 6 & None & $2(103)$ & $2(103)$ \\
\hline
\end{tabular}

${ }^{y}$ The number of field insensitive isolates is listed and the total number of tested isolates is given in parentheses. Isolates that showed normal growth at $5 \mu \mathrm{g} / \mathrm{ml}$ dimethachlon were considered resistant, whereas the completely inhibited ones were considered sensitive.

${ }^{\mathrm{z}}$ No samples were collected.

rape leaves (Table 4). There were no significant differences in lesion area caused by most of the dimethachlon-resistant and -sensitive isolates. However, one fieldinsensitive isolate (YC310R) and two laboratory-induced dimethachlon-resistant isolates $\left(\mathrm{CZ} 57 \mathrm{R}_{2}\right.$ and $\left.\mathrm{CZ57 \textrm {R } _ { 3 }}\right)$ produced smaller lesions on oilseed rape leaves than the other isolates.

Comparison of osmotic sensitivity. The laboratory-induced dimethachlonresistant isolates grew significantly more slowly than their wild-type sensitive parents on PDA plates amended with $\mathrm{NaCl}$ at each of the four concentrations, especially in the lower concentration (Fig. 2). However there were no significant differences $(P<0.05)$ in radial growth inhibition between the field-insensitive and -sensitive isolates at each of the $\mathrm{NaCl}$ concentrations.

\section{DISCUSSION}

During the course of our monitoring study, which was carried out during the growing seasons of 2006 to 2007, sensitive isolates of S. sclerotiorum, together with a small subpopulation of isolates with reduced sensitivity to dimethachlon, were found in two cities (Yancheng and Changzhou, Fig. 1) of Jiangsu Province. Five $S$. sclerotiorum isolates with reduced sensitivity to dimethachlon were detected among 1,066 isolates tested.

Control failures that could be attributed to resistance to dimethachlon have not yet been detected from the oilseed rape fields in Jiangsu Province where dimethachlon has been extensively used for more than 6 years. However, high levels of resistance to

Table 3. Sensitivity of the selected isolates that showed different resistance levels to dimethachlon and the dicarboximide fungicides iprodione and procymidione in Sclerotinia sclerotiorum

\begin{tabular}{|c|c|c|c|c|}
\hline Isolate $^{x}$ & Resistance phenotypes of the isolates & $\begin{array}{c}\text { EC }_{50} \text { values } \\
\text { of dimethachlon }(\mu \mathrm{g} / \mathrm{ml})^{\mathrm{y}}\end{array}$ & $\begin{array}{c}\text { EC }_{50} \text { values } \\
\text { of iprodione }(\mu \mathrm{g} / \mathrm{ml})^{\mathrm{y}}\end{array}$ & $\begin{array}{c}\mathbf{E C}_{50} \text { values } \\
\text { of procymidione }(\mu \mathrm{g} / \mathrm{ml})^{y}\end{array}$ \\
\hline CZ130 & Field dimethachlon-sensitive & $0.39 \mathrm{a}^{\mathrm{z}}$ & $0.21 \mathrm{a}$ & $0.19 \mathrm{a}$ \\
\hline CZ07 & Field dimethachlon-sensitive & $0.37 \mathrm{a}$ & $0.15 \mathrm{a}$ & $0.17 \mathrm{a}$ \\
\hline ZJ266 & Field dimethachlon-sensitive & $0.41 \mathrm{a}$ & $0.24 \mathrm{a}$ & $0.16 \mathrm{a}$ \\
\hline ZJ281 & Field dimethachlon-sensitive & $0.27 \mathrm{a}$ & $0.16 \mathrm{a}$ & $0.19 \mathrm{a}$ \\
\hline ZJ288 & Field dimethachlon-sensitive & $0.40 \mathrm{a}$ & $0.17 \mathrm{a}$ & $0.16 \mathrm{a}$ \\
\hline CZ94 & Field dimethachlon-sensitive & $0.38 \mathrm{a}$ & $0.18 \mathrm{a}$ & $0.16 \mathrm{a}$ \\
\hline CZ57 & Field dimethachlon-sensitive & $0.41 \mathrm{a}$ & $0.15 \mathrm{a}$ & $0.20 \mathrm{a}$ \\
\hline CZ87 & Field dimethachlon-sensitive & $0.32 \mathrm{a}$ & $0.27 \mathrm{a}$ & $0.26 \mathrm{a}$ \\
\hline YZ11 & Field dimethachlon-sensitive & $0.28 \mathrm{a}$ & $0.10 \mathrm{a}$ & $0.21 \mathrm{a}$ \\
\hline YZ12 & Field dimethachlon-sensitive & $0.37 \mathrm{a}$ & $0.16 \mathrm{a}$ & $0.12 \mathrm{a}$ \\
\hline HA84 & Field dimethachlon-sensitive & $0.23 \mathrm{a}$ & $0.21 \mathrm{a}$ & $0.22 \mathrm{a}$ \\
\hline YC312 & Field dimethachlon-insensitive & $4.42 \mathrm{c}$ & $4.56 \mathrm{~d}$ & $3.47 \mathrm{~b}$ \\
\hline YC310 & Field dimethachlon-insensitive & $2.37 \mathrm{~b}$ & $2.25 \mathrm{~b}$ & $4.17 \mathrm{~b}$ \\
\hline YC334 & Field dimethachlon-insensitive & $2.99 \mathrm{~b}$ & $2.00 \mathrm{~b}$ & $2.48 \mathrm{~b}$ \\
\hline CZ162 & Field dimethachlon-insensitive & $2.88 \mathrm{~b}$ & $3.16 \mathrm{c}$ & $2.45 \mathrm{~b}$ \\
\hline CZ138 & Field dimethachlon-insensitive & $3.12 \mathrm{~b}$ & $3.00 \mathrm{c}$ & $2.61 \mathrm{~b}$ \\
\hline ZJ288-1 & Laboratory-induced dimethachlon-resistant & $92.37 \mathrm{e}$ & $>100 \mathrm{f}$ & $220.39 \mathrm{e}$ \\
\hline ZJ288-2 & Laboratory-induced dimethachlon-resistant & $86.83 \mathrm{~d}$ & $>100 \mathrm{f}$ & $182.81 \mathrm{~d}$ \\
\hline ZJ288-3 & Laboratory-induced dimethachlon-resistant & $67.99 \mathrm{c}$ & $>100 \mathrm{f}$ & $462.54 \mathrm{~g}$ \\
\hline CZ57-1 & Laboratory-induced dimethachlon-resistant & $166.40 \mathrm{~h}$ & $>100 \mathrm{f}$ & $143.84 \mathrm{c}$ \\
\hline CZ57-2 & Laboratory-induced dimethachlon-resistant & $151.72 \mathrm{~g}$ & $>100 \mathrm{f}$ & $515.19 \mathrm{~h}$ \\
\hline CZ57-3 & Laboratory-induced dimethachlon-resistant & $124.81 \mathrm{f}$ & $42.40 \mathrm{e}$ & $276.88 \mathrm{f}$ \\
\hline
\end{tabular}

x ZJ288-1, ZJ288-2, ZJ288-3, CZ57-1, CZ57-2, and CZ57-3 were the laboratory-induced dimethachlon-resistant mutants derived from field dimethachlonsensitive parental isolates ZJ288 and CZ57, respectively.

${ }^{\mathrm{y}} \mathrm{The} \mathrm{EC}_{50}$ values, defined as the concentration of fungicide that caused a reduction in colony diameter by $50 \%$, was estimated by linear regression of the log of the colony diameter versus the fungicide concentration.

${ }^{\mathrm{z}}$ Means followed by the same letter were not different according to Fisher's least significant difference (LSD) $(P=0.05)$. 
dimethachlon could be obtained by in vitro selection for resistance. Based on our knowledge, this is the first report on the occurrence of dimethachlon-insensitive isolates of S. sclerotiorum in Jiangsu Province of China. Similar studies also showed that the heavy use of dicarboximide fungicides has led to the development of $S$. homoeocarpa (from golf courses) and $S$. minor (from peanut) isolates insensitive to these fungicides $(21,45)$. However, another study reported that no dicarboximideresistant isolates were detected from a field where dicarboximide fungicides were extensively used and losses had sometimes been severe (19).

Genetic analysis of dicarboximide resistance has demonstrated that the resistance is usually conferred by a single locus (Daf1) $(12,13,15,29,30)$, although a second locus (Daf2) may also be involved in the resistance in laboratory-induced mutants (16). The disruption of $u b c l$ gene and an enhanced level of antioxidant enzymes could also cause dicarboximide resistance $(38,43,46)$. In light of recent studies on dicarboximide resistance, it was concluded that resistance mechanisms to dicarboximides in phytopathogenic fungi are complex (31). Consequently, it may be difficult to use molecular methods for detecting dicarboximide resistance in fungal populations (31). The presence of two resistance phenotypes of $S$. sclerotiorum in this study suggests that at least two different mechanisms of resistance may be involved: one that leads to a moderate resistance with RF $\approx 10$ (as shown in the field dimethachloninsensitive isolates) and another that leads to a high resistance with RF 197.6 to 483.7 (as shown in the laboratory-induced dimethachlon-resistant isolates). A recent study also reported two resistance phenotypes in Stemphylium vesicarium isolates from pear orchards that showed moderate resistance $(\mathrm{RF} \approx 10)$ and high resistance ( $\mathrm{RF}>100)$ to dicarboximides, similar to the two resistance phenotypes in this study (2). Although the dicarboximide fungicides, such as iprodione, procymidone, and vinclozolin, have been widely used all over the world, the mode of action of these fungicides is still not fully understood $(2,5,6,18,20,22,30,36,39)$. Dicarboximide fungicides are chemically different even if they have an analogous mode of antifungal action, which also supports the hypothesis that there is more than one mechanism of resistance.

Information on fitness of resistant mutants is useful in developing effective antiresistance strategies. If fitness costs are associated with fungicide resistance, the frequency of the resistant pathogen population will decline when the fungicide selection pressure is discontinued in a field. A previous study reported that $S$. minor isolates from peanut with in vitro resistance to iprodione and vinclozolin were pathogenic to peanut in field microplots and survived as well as a fungicide-sensitive field isolate (9); whereas another study showed that the virulence of the dicarboximide-resistant $S$. minor

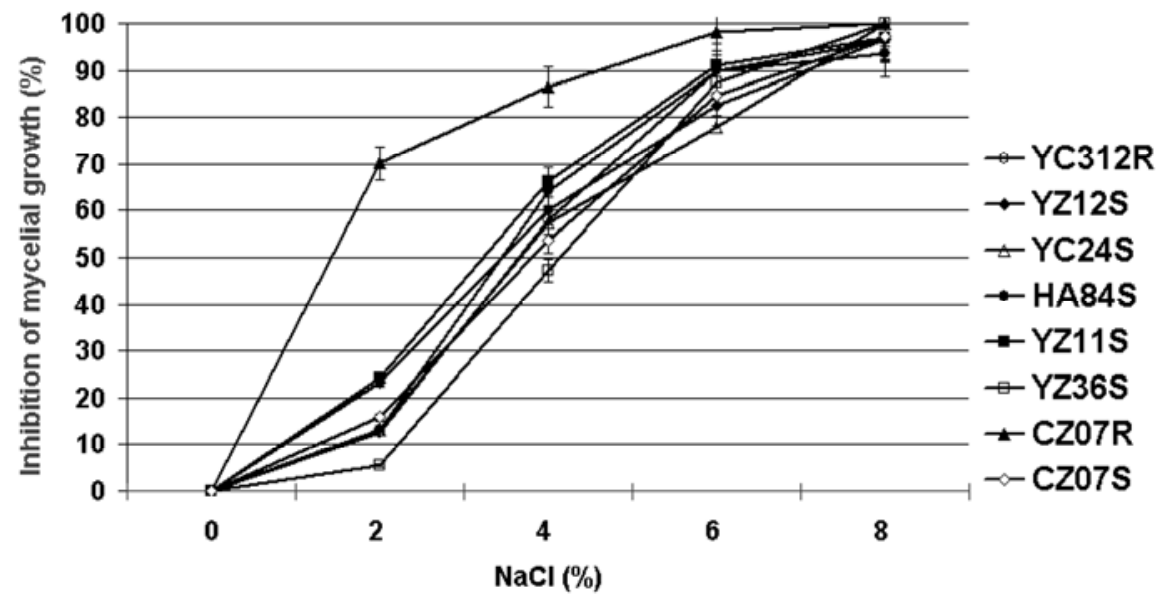

Fig. 2. Comparisons in osmotic sensitivity among laboratory-induced dimethachlon-resistant isolate CZ70R and its sensitive parent CZ70S, and field dimethachlon-insensitive isolate YC312R and other field dimethachlon-sensitive isolates YZ12S, YC24S, HA84S, YZ11S, and YZ36S. Data represent means $\pm \mathrm{SD}$ of the two experiments since there were no significant $(P=0.05)$ differences between the two experiments.

Table 4. Comparison of mycelial growth, sclerotial production, and pathogenicity among field dimethachlon-sensitive, field dimethachlon-insensitive, and laboratory-induced dimethachlon-resistant isolates

\begin{tabular}{|c|c|c|c|c|c|c|}
\hline \multirow[b]{2}{*}{ Isolates $^{v}$} & \multirow[b]{2}{*}{ Phenotype of sensitivity to dimethachlon } & \multicolumn{2}{|c|}{ Mycelial growth } & \multicolumn{2}{|c|}{ Sclerotia production } & \multirow[b]{2}{*}{$\begin{array}{l}\text { Lesion area } \\
\qquad\left(\mathrm{cm}^{2}\right)^{y}\end{array}$} \\
\hline & & $\begin{array}{l}\text { Mycelia radial } \\
\text { growth }(\mathbf{c m})^{w}\end{array}$ & $\begin{array}{c}\text { Dry weight } \\
\text { of mycelia }(\mathrm{g})^{\mathrm{x}}\end{array}$ & $\begin{array}{l}\text { Number } \\
\text { of sclerotia }\end{array}$ & $\begin{array}{l}\text { Dry weight per } \\
\text { sclerotium (mg) }\end{array}$ & \\
\hline YZ12S & Field dimethachlon-sensitive & $7.5 \mathrm{a}^{\mathrm{z}}$ & $0.65 \mathrm{a}$ & $25 \mathrm{a}$ & $2.2 \mathrm{~b}$ & $41.8 \mathrm{a}$ \\
\hline YZ11S & Field dimethachlon-sensitive & $7.4 \mathrm{a}$ & $0.65 \mathrm{a}$ & $24 \mathrm{a}$ & $2.2 \mathrm{~b}$ & $43.0 \mathrm{a}$ \\
\hline ZJ281S & Field dimethachlon-sensitive & $7.4 \mathrm{a}$ & $0.64 \mathrm{a}$ & $26 \mathrm{a}$ & $1.8 \mathrm{~b}$ & $41.4 \mathrm{a}$ \\
\hline CZ57S & Field dimethachlon-sensitive & $7.3 \mathrm{a}$ & $0.63 \mathrm{a}$ & $27 \mathrm{a}$ & $1.6 \mathrm{~b}$ & $41.1 \mathrm{a}$ \\
\hline YZ36S & Field dimethachlon-sensitive & $7.5 \mathrm{a}$ & $0.64 \mathrm{a}$ & $28 \mathrm{a}$ & $2.1 \mathrm{~b}$ & $40.7 \mathrm{a}$ \\
\hline CZ138R & Field dimethachlon-insensitive & $6.6 \mathrm{~b}$ & $0.59 \mathrm{ab}$ & $9 \mathrm{~b}$ & $6.0 \mathrm{a}$ & $45.3 \mathrm{a}$ \\
\hline CZ162R & Field dimethachlon-insensitive & $6.5 \mathrm{~b}$ & $0.53 \mathrm{ab}$ & $9 \mathrm{~b}$ & $6.9 \mathrm{a}$ & $42.7 \mathrm{a}$ \\
\hline YC312R & Field dimethachlon-insensitive & $6.4 \mathrm{~b}$ & $0.53 \mathrm{ab}$ & $5 \mathrm{~b}$ & $7.9 \mathrm{a}$ & $40.7 \mathrm{a}$ \\
\hline YC310R & Field dimethachlon-insensitive & $6.5 \mathrm{~b}$ & $0.60 \mathrm{ab}$ & $11 \mathrm{~b}$ & $7.8 \mathrm{a}$ & $12.7 \mathrm{c}$ \\
\hline YC334R & Field dimethachlon-insensitive & $6.6 \mathrm{~b}$ & $0.50 \mathrm{ab}$ & $12 \mathrm{~b}$ & $7.7 \mathrm{a}$ & $47.8 \mathrm{a}$ \\
\hline $\mathrm{ZJ} 281 \mathrm{R}_{1}$ & Laboratory-induced dimethachlon-resistant & $6.5 \mathrm{~b}$ & $0.50 \mathrm{ab}$ & $12 \mathrm{~b}$ & $7.3 \mathrm{a}$ & $41.0 \mathrm{a}$ \\
\hline $\mathrm{ZJ} 281 \mathrm{R}_{2}$ & Laboratory-induced dimethachlon-resistant & $6.6 \mathrm{~b}$ & $0.42 \mathrm{~b}$ & $11 \mathrm{~b}$ & $7.4 \mathrm{a}$ & $50.4 \mathrm{a}$ \\
\hline $\mathrm{ZJ} 281 \mathrm{R}_{3}$ & Laboratory-induced dimethachlon-resistant & $6.5 \mathrm{~b}$ & $0.45 \mathrm{ab}$ & $9 \mathrm{~b}$ & $7.2 \mathrm{a}$ & $40.7 \mathrm{a}$ \\
\hline $\mathrm{CZ} 7 \mathrm{R}_{1}$ & Laboratory-induced dimethachlon-resistant & $6.7 \mathrm{~b}$ & $0.55 \mathrm{ab}$ & $10 \mathrm{~b}$ & $6.9 \mathrm{a}$ & $42.0 \mathrm{a}$ \\
\hline $\mathrm{CZ} 57 \mathrm{R}_{2}$ & Laboratory-induced dimethachlon-resistant & $6.5 \mathrm{~b}$ & $0.55 \mathrm{ab}$ & $8 \mathrm{~b}$ & $7.2 \mathrm{a}$ & $22.8 \mathrm{~b}$ \\
\hline $\mathrm{CZ} 57 \mathrm{R}_{3}$ & Laboratory-induced dimethachlon-resistant & $6.4 \mathrm{~b}$ & $0.53 \mathrm{ab}$ & $9 \mathrm{~b}$ & $6.8 \mathrm{a}$ & $26.4 \mathrm{~b}$ \\
\hline
\end{tabular}

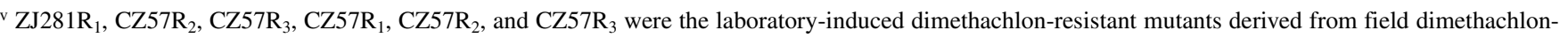
sensitive parental isolates $\mathrm{ZJ} 281$ and CZ57, respectively.

${ }^{w}$ The mycelial radial growth after 2 days of incubation on potato dextrose agar (PDA) at $25^{\circ} \mathrm{C}$ in a growth chamber ( $12 \mathrm{~h}$ photoperiod) was measured, and the two perpendicular diameters of each fungal colony were averaged.

${ }^{x}$ A fresh mycelial plug of each isolate from colony margins was transferred into a flask containing $100 \mathrm{ml}$ of potato dextrose (PD) broth for shake culture $\left(120 \mathrm{r} / \mathrm{min}, 25^{\circ} \mathrm{C}\right)$ for 2 days, and the dry weight of the mycelia was measured to further compare mycelial growth.

${ }^{\mathrm{y}}$ Lesions were measured as the mean of two diameters measured at right angles to one another after 4 days at $22^{\circ} \mathrm{C}$ under a 16 -h photoperiod and $\approx 80 \%$ relative humidity.

${ }^{\mathrm{z}}$ Means followed by the same letter were not different according to Fisher's least significant difference (LSD) test $(P=0.05)$. 
strains from lettuce declined over time (19). Also, laboratory-induced iprodioneresistant Alternaria isolates exhibited greater osmotic sensitivity than sensitive isolates, and this high osmotic sensitivity was considered to be responsible for lower pathogenicity and fitness of the resistant isolates than those of their sensitive counterparts $(18,30)$.

During the course of this study, five field isolates of S. sclerotiorum were identified as having reduced sensitivity to dimethachlon, and these isolates showed comparable mycelial growth and pathogenicity to the wild-type sensitive isolates. The same case occurred in the laboratoryinduced dimethachlon-resistant isolates. In addition, sclerotial production was lower in all laboratory-induced dimethachlonresistant mutants and the field dimethachlon-insensitive isolates, indicating that the resistance mutation or mutations might presumably be involved in the reduced sclerotial production. We also found that these five field dimethachlon-insensitive $S$. sclerotiorum isolates did not show higher osmotic sensitivity than field dimethachlonsensitive isolates, and they retained high virulence on oilseed rape. These results suggest that the field dimethachloninsensitive isolates may have sufficient parasitic fitness to compete with the field dimethachlon-sensitive isolates in the field. However, laboratory-induced dimethachlon-resistant isolates had high levels of resistance to dicarboximide fungicides and showed higher osmotic sensitivity compared to their original sensitive parents.

In this study, both laboratory-induced dimethachlon-resistant isolates and field dimethachlon-insensitive isolates showed stable dimethachlon resistance/insensitivity, and there was positive cross-resistance between dimethachlon and other well-known dicarboximide fungicides in S. sclerotiorum. Because these studies yielded a high frequency of laboratory-resistance in $S$. sclerotiorum and a low frequency of field insensitivity, appropriate precautions against resistance development in natural populations should be taken. China now is facing the challenge of finding alternative fungicides to control Sclerotinia stem rot in oilseed rape crops. Moreover, in order to better understand dimethachlon resistance, the resistance mechanism in both laboratory-induced dimethachlon-resistant isolates and field dimethachlon-insensitive isolates is currently under further investigation in our laboratory.

\section{ACKNOWLEDGMENTS}

This study was sponsored by the Science and Technology Support Programs from Jiangsu Province and the Ministry of Science and Technology (NO.2006CB101907, nyhyzx07-054, BE2006304, 2006AA10A211 and 2008AA102414).

\section{LITERATURE CITED}

1. Abawi, G. S., and Grogan, R. G. 1979. Epidemiology of diseases caused by Sclerotinia species. Phytopathology 69:899-904.
2. Alberoni, G., Collina, M., Pancaldi, D., and Brunelli, A. 2005. Resistance to dicarboximide fungicides in Stemphylium vesicarium of Italian pear orchards. Eur. J. Plant Pathol. 113:211-219.

3. Anonymous. 1975. Sclerotinia disease of oilseed crops. Oilcrop Research Institute, Chinese Academy of Sciences, Agriculture Press, Beijing, China.

4. Bardin, S. D., and Huang, H. C. 2001. Research on biology and control of Sclerotinia diseases in Canada. Can. J. Plant Pathol. 23:88-98.

5. Beever, R. E., and Brien, H. M. R. 1983. A survey of resistance to the dicarboximide fungicides in Botrytis cinerea. N.Z. J. Agric. Res. 26:391-400.

6. Biggs, A. R. 1994. Mycelial growth, sporulation, and virulence to apple fruit of Alternaria alternata isolates resistant to iprodione. Plant Dis. 78:732-735.

7. Boland, G. J., and Hall, R. 1994. Index of plant hosts of Sclerotinia sclerotiorum. Can. J. Plant Pathol. 16:93-108.

8. Bolton, D. M., Thomma, P. H. J. B., and Nelson, D. B. 2006. Sclerotinia sclerotiorum (Lib.) de Bary: Biology and molecular traits of a cosmopolitan pathogen. Mol. Plant Pathol. 7(1):1-16.

9. Brenneman, T. B., Phipps, P. M., and Stipes, R. J. 1987. Sclerotinia blight of peanut: Relationship between in vitro resistance and field efficacy of dicarboximide fungicides. Phytopathology 77:1028-1032.

10. Brent, K. J. 1988. Monitoring for fungicide resistance. Pages 9-11 in: Fungicide Resistance in North America. C. J. Delp, ed. American Phytopathological Society, St. Paul, MN.

11. Christian, T., Christopher, J. W., Jolanta, J. B., Rikard, H., and Berndt, G. 2001. Suppression of Sclerotinia sclerotiorum apothecial by the soil bacterium Serratia plymuthica: Identification of a chlorinated macrolide as one of the causal agents. Soil Biol. Biochem. 33:18171826.

12. Cui, W., Beever, R. E., Parkes, S. L., and Templeton, M. D. 2004. Evolution of an osmosensing histidine kinase in field strains of Botryotinia fuckeliana (Botrytis cinerea) in response to dicarboximide fungicide usage. Phytopathology 94:1129-1135.

13. Cui, W., Beever, R. E., Parkes, S. L., Weeds, P. L., and Templeton, M. D. 2002. An osmosensing histidine kinase mediates dicarboximide fungicide resistance in Botryotinia fuckeliana (Botrytis cinerea). Fungal Genet. Biol. 36:187198.

14. Dai, F. M., Xu, T., Wolf, G. A., and He, Z. H. 2006. Physiological and molecular features of the pathosystem Arabidopsis thaliana L. and Sclerotinia sclerotiorum Libert. J. Integr. Plant Biol. 48(1):44-52.

15. Faretra, F., and Pollastro, S. 1991. Genetic basis of resistance to benzimidazole and dicarboximide fungicide in Botryotinia fuckeliana (Botrytis cinerea). Mycol. Res. 95:943-951.

16. Faretra, F., and Pollastro, S. 1993. Isolation, characterization and genetic analysis of laboratory mutants of Botryotinia fuckeliana resistant to the phyenylpyrrole fungicide CGA 173506. Mycol. Res. 97:620-624.

17. Hu, X. J., Roberts, D. P., Jiang, M. L., and Zhang, Y. B. 2005. Decreased incidence of disease caused by Sclerotinia sclerotiorum and improved plant vigor of oilseed rape with $B a$ cillus subtilis Tu-100. Appl. Microbiol. Biotechnol. 68:802-807.

18. Huang, R., and Levy, Y. 1995. Characterization of iprodione-resistant isolates of Alternaria brassicicola. Plant Dis. 79:828-833.

19. Hubbard, J. C., Subbarao, K. V., and Koike, S. T. 1997. Development and significance of dicarboximide resistance in Sclerotinia minor isolates from commercial lettuce fields in Cali- fornia. Plant Dis. 81:148-153.

20. Iacomi-Vasilescu, B., Avebot, H., BatailléSimoneau, N., Laurent, E., Guénard, M., and Simoneau, P. 2004. In vitro fungicide sensitivity of Alternaria species pathogenic to crucifers and identification of Alternaria brassicicola field isolates highly resistant to both dicarboximides and phenylpyrroles. Crop Prot. 23:481-488.

21. Jo, Y.-K., Niver, A. L., Rimelspach, J. W., and Boehm, M. J. 2006. Fungicide sensitivity of Sclerotinia homoeocarpa from golf courses in Ohio. Plant Dis. 90:807-813.

22. Katan, T. 1982. Resistance to 3, 5dichlorophenyl-N-cyclicimide (dicarboximide) fungicides in the grey mould pathogen Botrytis cinerea on protected crops. Plant Pathol. 31:133-141.

23. Leroux, P., Fritz, R., Debieu, D., Albertini, C., Lanen, C., Bach, J., Gredt, M., and Chapeland, F. 2002. Mechanisms of resistance to fungicides in field strains of Botrytis cinerea. Pest Manag. Sci. 58:876-888.

24. Letham, D. B., Huett, D. O., and Trimboli, D. S. 1976. Biology and control of Sclerotinia sclerotiorum in cauliflower and tomato crops in coastal New South Wales. Plant Dis. Rep. 60:286-289.

25. Li, H. X., Lu, Y. J., Zhou, M. G., and Wang, X. F. 2003. Mutation in $\beta$-tubulin of Sclerotinia sclerotiorum conferring resistance to carbendazim in rapeseed field isolate. Chin. J. Oil Crop Sci. 25(2):56-60.

26. Li, Y. J., Chen, H. M., and Zhou, L. 2003. Experiment on mixed pesticides for controlling tobacco brown spot and MyzusPersicae (Sulzer). Chin. J. Tobacco Sci. 10(2):45-47.

27. Lorenz, G. 1988. Discarboximide fungicides: History of resistance development and monitoring methods. Pages 45-51 in: Fungicide Resistance in North America. IV Dicarboximide Fungicides. C. J. Delp, B. R. Delp, T. M. Fortii, H. V. Morton, and C. M. Smith, eds. American Phytopathological Society, St Paul, MN.

28. Lu, G. 2003. Engineering Sclerotinia sclerotiorum resistance in oilseed crops. Afr. J. Biotechnol. 2:509-516.

29. Ma, Z., Lei, Y., Luo, Y., and Michailides, J. T. 2007. Sequence variation in the twocomponent histidine kinase gene of Botrytis cinerea associated with resistance to dicarboximide fungicides. Pestic. Biochem. Phys. 88:300-306

30. Ma, Z., and Michailides, J. T. 2004. Characterization of iprodione-resistant Alternaria isolates from pistachio in California. Pestic. Biochem. Phys. 80:75-84.

31. Ma, Z., and Michailides, J. T. 2005. Advances in understanding molecular mechanisms of fungicide resistance and molecular detection of resistant genotypes in phytopathogenic fungi. Crop Prot. 24:853-863.

32. Matheron, M. E., and Porchas, M. 2004. Activity of boscalid, fenhexamid, fluazinam, fludioxonil, and vinclozolin on growth of Sclerotinia minor and $S$. sclerotiorum and development of lettuce drop. Plant Dis. 88:665-668.

33. Mueller, D. S., Dorrance, A. E., Derksen, R C., Ozkan, E., Kurle, J. E., Grau, C. R., Gaska, J. M., Hartman, G. L., Bradley, C. A., and Pedersen, W. L. 2002. Efficacy of fungicides on Sclerotinia sclerotiorum and their potential for control of Sclerotinia stem rot on soybean. Plant Dis. 86:26-31.

34. Pan, Y. L. 1998. The resistance of Sclerotinia sclerotiorum of rape to carbendazim and its management. Jiangsu J. Agric. Sci. 14(3):159163.

35. Pieckenstain, F. L., Garriz, A., Chornomaz, E. M., Sanchez, D. H., and Ruiz, O. A. 2001. The effect of polyamine biosynthesis inhibition on growth and differentiation of the phytopatho- 
genic fungus Sclerotinia sclerotiorum. Anton. Leeuw. 80:245-253.

36. Pommer, E. H., and Lorenz, G. 1995. Dicarboximide fungicides. Pages 99-118 in: Modern Selective Fungicides - Properties, Applications, Mechanisms of Action. 2nd ed. H. Lyr, ed. Gustav Fischer, New York.

37. Purdy, L. H. 1979. Sclerotinia sclerotiorum: History, disease, and symptomatology, host range, geographic distribution, and impact. Phytopathology 69:875-880.

38. Ramesh, M. A., Laidlaw, R. D., Durrenberger, F., Orth, A. B., and Kronstad, J. W. 2001. The cAMP signal transduction pathway mediates resistance to dicarboximide and aromatic hydrocarbon fungicides in Ustilago maydis. Fungal Genet. Biol. 32:183-193.

39. Raposo, R., Gomez, V., Urrutia, T., and Melgarejo, P. 2000. Fitness of Botrytis cinerea associated with dicarboximide resistance. Phytopathology 90:1246-1249.

40. Sarma, B. K., Ameer, B. S., Singh, D. P., and Singh, U. P. 2007. Use of non-conventional chemicals as an alternative approach to protect chickpea (Cicer arietinum) from Sclerotinia stem rot. Crop Prot. 26:1042-1048.
41. Shi, Z. Q., Zhou, M. G., and Ye, Z. Y. 2000. Resistance of Sclerotinia Sclerotiorum to carbendazim and dimethachlon. Chin. J. Oil Crop Sci. 22(4):54-57.

42. Shi, Z. Q., Zhou, M. G., Ye, Z. Y., Shi, J. R., Chen, H. G., and Wang, Y. Z. 2000. Resistance monitoring of Sclerotinia sclerotiorum to carbendazim. J. Jiangsu Agric. Sci. 16(4):226229.

43. Sisler, H. D. 1988. Dicarboximide fungicides: Mechanisms of action and resistance. Page 52 in: Fungicides Resistance in North America. IV. Dicarboximide fungicides. C. J. Delp, B. R. Delp, T. M. Fortii, H. V. Morton, and C. M. Smith, eds. American Phytopathological Society, St. Paul, MN

44. Smith, F. D., Phipps, P. M., and Stipes, R. J. 1991. Agar plate, soil plate, and field evaluation of fluazinam and other fungicides for control of Sclerotinia minor on peanut. Plant Dis. 75:1138-1143.

45. Smith, F. D., Phipps, P. M., Stipes, R. J., and Brenneman, T. B. 1995. Significance of insensitivity of Sclerotinia minor to iprodione in control of Sclerotinia blight of peanut. Plant Dis. 79:517-523.
46. Steel, C. C., and Nair, N. G. 1993. The physiological basis of resistance to the dicarboximide fungicide iprodione in Botrytis cinerea. Pestic. Biochem. Phys. 47:60-68.

47. Suty, A., and Stenzel, K. 1999. Iprovalicarbsensitivity of Phytophthora infestans and Plasmopara viticola: Determination of baseline sensitivity and assessment of the risk of resistance. Pflanzenscbutz-Nachr. Bayer 52:171-182.

48. Sylvester-Bradley, R., and Donald, C. M 1984. A code for stages of development in oilseed rape (Brassica napus L.) Aspect. Appl. Biol. 6:399-419.

49. Zhang, X. L., Sun, X. M., and Zhang, G. F. 2003. Preliminary report on the monitoring of the resistance of sclerotinia libertinia to carbendazim and its internal management. Chin. J. Pest. Sci. Adm. 24(6):18-22.

50. Zhao, J., and Meng, J. 2003. Detection of loci controlling seed glucosinolate content and their association with Sclerotinia resistance in Brassica napus. Plant Breed. 122:19-23.

51. Zhou, B. W., and Luo, Q. 1994. Rapeseed Diseases and Control. China Commerce Publishing Co., Beijing, China. (in Chinese) 